Casos Clínicos

Arch. Esp. Urol. 2009; 62 (9): 747-751

\section{TERATOMA QUÍSTICO MADURO TESTICULAR (QUISTE DERMOIDE). APORTACIÓN DE UN CASO Y REVISIÓN DE LA LITERATURA.}

\author{
P. Garrido Abad, L.M. Herranz Fernández, M. Jiménez \\ Gálvez, C. Suárez Fonseca, D. Santos Arrontes, \\ S. Nieto Llanos' y M. Fernández Ariona.
}

Servicio de Urología y Servicio de Anatomía Patológica?. Hospital del Henares. Coslada. Madrid. España.

Resumen.- OBJETIVOS: Aportamos un caso de un teratoma quístico maduro testicular (quiste dermoide) en su variante pseudopilomatrixoma.

MÉTODOS: Paciente de 53 años con una masa testicular izquierda no dolorosa al que, tras la realización de orquiectomía radical, se diagnostica de teratoma quístico maduro testicular (quiste dermoide) en su variante pseudopilomatrixoma. Estudio de extensión y marcadores tumorales negativos. El paciente se encuentra libre de enfermedad tras 6 meses de seguimiento.

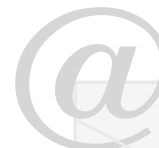

\section{CORRESPONDENCIA}

Pablo Garrido Abad

Arcos de la Frontera, 27 - 2A

28023. Madrid. (España)

pgabad@hotmail.com

Trabajo recibido: 20 de abril 2009.
RESULTADOS: El teratoma testicular es un tumor compuesto por células derivadas de las tres hojas embrionarias lectodermo, endodermo y mesodermo). Aparecen a cualquier edad, siendo mucho más incidentes en la infancia, donde llegan a representar hasta un 30\% de todos los tumores. En la edad adulta es mucho menos prevalente, llegando sólo a representar un $7 \%$ de todos los tumores germinales del testículo.

CONCLUSIONES: Los casos prepuberales son casi invariablemente de carácter benigno, independientemente de su histología. Sin embargo, los casos que aparecen tras la pubertad presentan potencial maligno, incluso si es histológicamente puro. El teratoma quístico maduro lquiste dermoide) representa la única excepción a esta regla, ya que no se ha publicado ninguna degeneración maligna de este tipo de tumores.

Palabras clave: Teratoma. Testículo. Tumor.

Summary.- OBJECTIVES: Case report of a mature cystic teratoma (dermoid cyst), pseudopilomatrixoma like variant.

METHOD: 53-year old patient with a left testicular mass, diagnosed as mature cystic teratoma of the testis /dermoid cyst), the pilomatrixoma-like variant, after radical orchiectomy. Tumoral markers were negative and no extension was observed. The patient is disease-free after 6 months follow-up.

RESULTS: Testicular teratoma is a tumour composed of cells derived from the three embryonic layers lectoderm, endoderm and mesoderm). They can appear at any age, but they are much more prevalent in childhood. They appear at any age, but are much more common in childhood, where they comprise up to $30 \%$ of all tumours. They are much less prevalent in adults, representing only $7 \%$ of all testicular germ cell tumours

CONCLUSIONS: Prepubertal cases are invariably benign in nature, regardless of their histology. However, cases which appear after puberty are potentially malignant, even if histologically pure. Mature cystic teratoma (dermoid cyst) is the only exception to this rule, as no malignant degeneration of these types of tumours has been published.

Keywords: Teratoma. Testicle. Tumour.

\section{INTRODUCCIÓN}

Los teratomas testiculares son un tipo de tumor compuesto típicamente por varios tejidos que representan las diferentes capas germinales (endodermo, mesodermo y ectodermo). 
Aparecen a cualquier edad, siendo mucho más incidentes en la infancia, donde llegan a representar hasta un $30 \%$ de todos los tumores. En la edad adulta es mucho menos prevalente, llegando sólo a representar un $7 \%$ de todos los tumores germinales del testículo (1).

Presenta diferentes variantes en función de sus componentes histológicos. Sin embargo, el quiste dermoide testicular puede según diversos autores ser incluido dentro de los teratomas testiculares (teratoma quístico maduro), - bien, más recientemente ser considerado como una entidad independiente $(2,3)$.

\section{CASO CLÍNICO}

Paciente varón de 53 años de edad que acude a la consulta de urología por masa testicular izquierda. En la exploración física destaca la palpación de una lesión en polo superior de testículo izquierdo, de consistencia firme y no dolorosa.

Se realiza ecografía testicular donde se describe un área de $2 \times 2 \mathrm{cms}$, de ecogenicidad heterogénea, con múltiples zonas quísticas, sugerente de posible neoplasia. Los marcadores tumorales son negativos. Ante la alta sospecha de tumor testicular, decidimos realización de orquiectomía radical izquierda.

En el estudio histológico de la pieza quirúrgica, macroscópicamente se describe una tumoración bien delimitada, de $1.8 \mathrm{cms}$. de diámetro, color amarillento y consistencia pétrea. Microscópicamente se encuentra constituido por escamas córneas y nidos de epitelio escamoso en el que se reconocen las células epiteliales "en sombra". La mayor parte de este material se encuentra calcificado y osificado, observándose alrededor de la lesión, reacción fibrosa que determina la formación

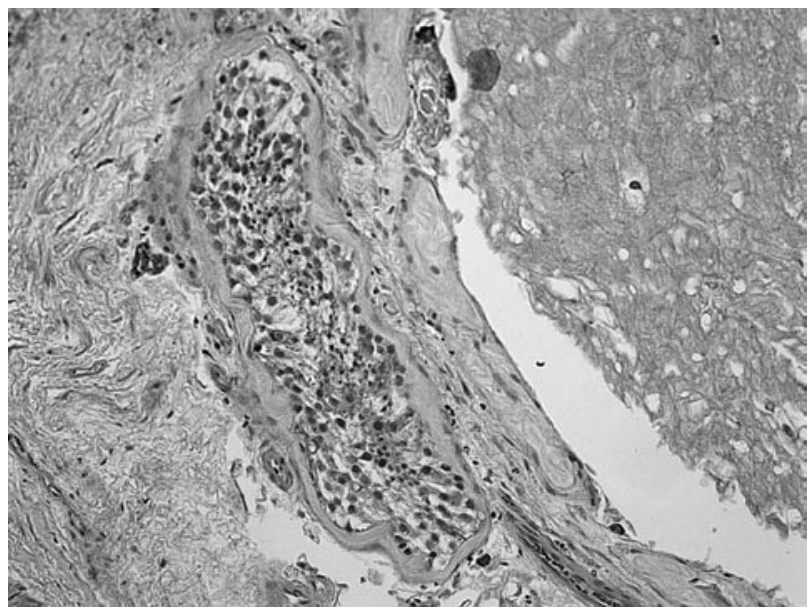

FIGURA 1. (H-E, 4OX): Área de pilomatrixoma junto a túbulo seminífero atrapado. de una pseudocápsula que delimita el tumor. En esta pseudocápsula fibrosa se identifican focalmente haces de músculo liso. El parénquima testicular muestra un moderado edema intersticial. El epidídimo y el cordón espermático no presentan alteraciones histológicas relevantes. El diagnóstico anatomo-patológico informa de quiste dermoide testicular (teratoma maduro testicular), variante pseudopilomatrixoma (Figuras 1 y 2).

Tras la orquiectomía se realiza estudio de extensión mediante radiografía de tórax y TAC abdominopélvico, sin evidenciarse extensión sistémica de la enfermedad. Los marcadores tumorales testiculares (alfa-fetoproteína, beta-HCG y LDH) son negativos.

El paciente se encuentra asintomático y libre de enfermedad en la actualidad tras 6 meses de seguimiento.

\section{DISCUSIÓN}

El cáncer testicular es la neoplasia maligna más frecuente en varones de 20 a 35 años y supone, aproximadamente, el $1 \%$ de todas las neoplasias de los varones. Los tumores de células germinales (TCG) del testículo pueden clasificarse en dos grupos principales: seminomatosos y no seminomatosos. Las histologías no seminomatosas incluyen carcinoma de células embrionarias, tumor del saco vitelino, coriocarcinoma, y teratoma. En la mayoría de los casos se identifica más de un patrón histológico, siendo la combinación más frecuente el hallazgo de carcinoma embrionario, tumor del saco vitelino y teratoma (4).

Un teratoma es un tipo de tumor de origen embrionario. El término teratoma deriva del griego "tumor monstruoso". Son un tipo de tumor típicamente compuesto por varios tejidos que representan las diferentes capas ger-

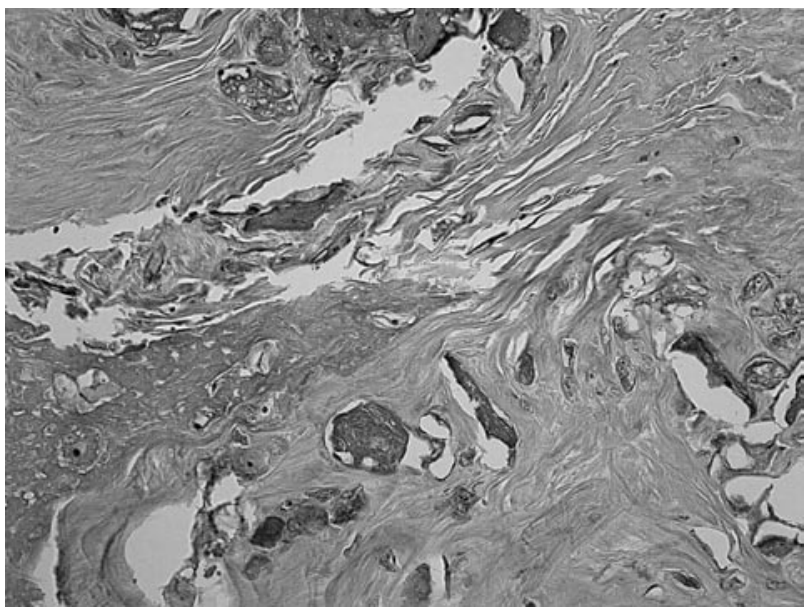

FIGURA 2. (H-E, 4OX): Áreas de pilomatrixoma. 
minales. Contienen células derivadas de las tres hojas embrionarias (ectodermo, endodermo y mesodermo) que se diferencian para formar tejidos de tipo somático típicos del desarrollo adulto o embrionario. Los elementos ectodérmicos incluyen epitelio escamoso y tejido neuronal. Los derivados del endodermo son células del epitelio gastrointestinal, respiratorio y otras glándulas mucosas. Los elementos derivados del mesodermo incluyen hueso, cartílago y músculo $(5,6)$.

Se reconocen 4 subtipos de teratomas testiculares según su composición histológica:

1) Maduros: (5-10\%) Compuestos exclusivamente por tejidos somáticos maduros bien diferenciados. Típicamente contienen estructuras derivadas de las tres hojas germinales. A pesar de que su apariencia histológica recuerda a los tejidos adultos, con frecuencia se encuentra aumento de celularidad, ligera a moderada atipia citológica, con aneuplioidia, y ocasional actividad mitótica tanto en los tejidos mesenquimales como en los epiteliales, pero estos cambios no justifican su categorización como inmaduros (7).

2) Inmaduros: (20-30\%) Su inmadurez viene determinada por la presencia de tejidos que no pueden ser reconocidos como elementos adultos normales. Contienen elementos incompletamente diferenciados o embrionarios, además de cantidades variables de elementos maduros. Son componentes frecuentes los elementos neuroectodérmicos fetales, glándulas mucosas embrionarias, cartílago y elementos mesenquimales inmaduros. Las mitosis pueden ser muy numerosas (6).

3) Con áreas malignas: Son teratomas, generalmente inmaduros en adultos, que se caracterizan por el sobrecrecimiento de una segunda neoplasia maligna de células no germinales, esto es un sarcoma (rabdomiosarcoma, condrosarcomas), un carcinoma (adenocarcinoma y carcinoma epidermoide) o ambos. La presencia de este componente maligno en un teratoma en el testículo no altera el pronóstico del paciente, pero si las metástasis contienen carcinoma o sarcoma derivado del teratoma, el pronóstico es infausto.

\section{4) Variantes monodérmicas:}

a. Carcinoides: Raros (0.17\%). Idénticas características que los fumores carcinoides de otras localizaciones. Tres cuartas partes aparecen de forma testicular pura; el resto en el seno de teratomas. La mayoría tienen comportamiento benigno, apareciendo metástasis en menos del $10 \%$ de los casos $(6)$.

b. Tumor Neuroectodérmico Primitivo (PNET): Extremadamente rara su presencia de forma pura, siendo la mayoría un mínimo componente de tumores germinales mixtos (6).

c. Otros teratomas monodérmicos.
El teratoma testicular es un tumor que aparece en cualquier edad, con una media de presentación de 29 años. Presenta una distribución simétrica en cuanto a la lateralidad, con algún caso de afectación sincrónica bilateral (8). Se ha descrito su asociación hasta en un $7 \%$ con historia de criptorquidia ipsilateral previa. Representa el $20-30 \%$ de los tumores de la infancia. En la literatura se describe el teratoma maduro testicular prepuberal como una tumoración enteramente benigna sin afectación retroperitoneal o visceral.

Sin embargo, en el adulto, el teratoma puro maduro representa sólo del 2.7 al $3 \%$ de los tumores del testículo, presentando afectación metastásica variable. Según la mayoría de estudios, el teratoma puro testicular se presenta en estadíos iniciales (I-IIA/B) en el $44-79 \%$ de los pacientes. En un $20-56 \%$ de los pacientes se presenta en estadíos clínicamente avanzados (IIC-IV) con metástasis. Sin embargo otros autores estiman que la incidencia de enfermedad avanzada al inicio del diagnóstico es de hasta el $60 \%$. La incidencia de metástasis retroperitoneales en pacientes con enfermedad en estadío clínico I es, aproximadamente del $30 \%$, y puede aumentar hasta el $75 \%$ en pacientes con enfermedad en estadío clínico IIA. Incluso en pacientes adecuadamente seleccionados con teratoma testicular puro, aproximadamente el $20 \%$ de ellos tendrá una recurrencia durante el seguimiento, siendo el retroperitoneo la localización más frecuente de la recurrencia, seguido de los pulmones o la elevación aislada de los marcadores tumorales séricos $(4,9-11)$.

Tanto los teratomas maduros como inmaduros pueden sufrir malignización hacia carcinoma o sarcoma, con posibilidad de invasión vascular asociada. Poseen potencial para metastatizar en los ganglios linfáticos retroperitoneales o sistémicamente en otros órganos. Las metástasis pueden contener tanto elementos teratomatosos como no teratomatosos. Debido a su apariencia histológica benigna se le considera un tumor testicular germinal no seminomatoso de baja agresividad. De hecho, la presencia de componente teratomatoso en el seno de una tumoración testicular germinal mixta no seminomatosa, especialmente si ésta supera el $50 \%$, confiere una menor incidencia de afectación metastásica (12). El teratoma, aunque es histológicamente benigno, tiene un potencial biológico variado. La capacidad del teratoma de presentar crecimiento agresivo local, diseminación a distancia y transformación en una neoplasia maligna somática pone de relieve la imprevisibilidad de este tumor. Por todo ello debe considerarse como una tumoración maligna.

Existe controversia sobre la terminología "quiste dermoide" testicular. Diversos autores engloban el quiste dermoide dentro de los teratomas testiculares. Sin embargo, otros autores postulan que el quiste dermoide testicular se puede considerar una entidad aparte de los teratomas maduros, con una historia natural benigna. Para ello, describen ciertas características que pueden servir para la realización del diagnóstico diferencial 
entre el quiste dermoide y el teratoma testicular. Los teratomas testiculares maduros presentan componentes sólidos y quísticos frente a la sola presencia de material quístico en el quiste dermoide. La presencia de pelo intralesional es típica sólo del quiste dermoide, apareciendo de hecho en más de la mitad de todos ellos. El teratoma maduro se relaciona hasta en un 80 $88 \%$ de los casos con intratubular germ cell neoplasia of the unclassified type (IGCNU), lo cual nunca ocurre en los quistes dermoides. Finalmente destacar la mayor presencia de atipia y elevada actividad mitótica en los teratomas maduros testiculares, que no ocurren en los quistes dermoides $(2,3)$.

Se han publicado menos de 20 casos de quiste dermoide testicular con histología y seguimiento adecuados en la literatura mundial, y tan sólo dos en su variante pilomatrixoma $(2,13)$. En estos últimos es típica la presencia de células epiteliales escamosas "en sombra", con zonas de calcificación y osificación.

En ningún caso se ha observado la presencia de afectación metastásica. Se recomienda la especificación de la presencia de quiste dermoide testicular, ya que, aunque puede representar un teratoma en sí mismo, modifica de forma importante tanto el pronóstico como la actitud terapeútica y el seguimiento. Se pueden evitar así protocolos de seguimiento demasiado estrictos y duraderos para los pacientes, así como la realización de LRP innecesarias (2).

La ecografía testicular suele ser muy útil, ya que presenta una gran sensibilidad en la detección de masas escrotales y su localización. Sin embargo en muchas ocasiones no es fácil diferenciar entre tumores benignos, malignos o procesos inflamatorios. No existe un patrón ecográfico definido de los teratomas testiculares. Con frecuencia en el estudio ecográfico los teratomas pueden tener el aspecto se zonas quísticas con tabiques y zonas sólidas hiperecogénicas interpuestas. La presencia de calcificaciones en el tumor es otro hallazgo ecográfico útil asociado con los teratomas. Sin embargo, en la actualidad, no somos capaces de diagnosticar un teratoma exclusivamente con ecografía.

Con otras técnicas de imagen como la TAC o la RMN podemos obtener mayor información ya sea a nivel local o a distancia. Sin embargo, el diagnóstico debe siempre confirmarse con el estudio anatomo-patólogico.

Los marcadores tumorales serológicos como la beta-gonadotrofina coriónica (HCG) y la alfa-fetoproteína (AFP) suelen ser negativos en casos de teratoma maduro (5). La concentración sérica de alfa-proteína (AFP) también es útil para diferenciar los teratomas de los tumores del saco vitelino. Los teratomas no se tiñen positivamente para AFP mediante inmunohistoquímica, y no se ha encontrado elevación de la concentración sérica de AFP en los pacientes que tienen estos tumores.
El hallazgo clínico más frecuente del teratoma testicular, es la aparición de una masa testicular indolora, la cual puede confundirse en ocasiones con un hidrocele, debido a su transiluminación parcialmente positiva (5).

El tratamiento estándar de todos los tumores testiculares de células germinales en los adultos es la orquiectomía radical, realizada mediante una vía de abordaje inguinal.

Sin embargo, en los pacientes con teratoma puro en estadíos iniciales, existe controversia sobre el abordaje del retroperitoneo Las opciones terapeúticas para estos pacientes incluyen disección de los ganglios linfáticos retroperitoneales (LRP) o vigilancia.

En los pacientes en estadío avanzado, los teratomas testiculares clásicamente han sufrido la regresión de otros elementos de células germinales no seminomatosos, "tumor agotado". Los marcadores tumorales séricos están elevados con frecuencia, y también con frecuencia hay TCG viables (carcinoma de células embrionarias o tumor del saco vitelino) en las localizaciones metastásicas. Por todo ello, y a pesar de la quimioresistencia conocida del teratoma, a estos pacientes se les suele tratar inicialmente con quimioterapia para mantener la LRP en segundo lugar si persiste masa residual (4). Otros autores prefieren asumir que las metástasis son de la misma estirpe teratomatosa y no aplican tratamiento quimioterápico previo (14).

Las tasas de supervivencia después del tratamiento con seguimiento tras la orquiectomía es del 95-100\%, siendo comparable a las de las LRP primaria. Entre los factores que pueden predecir una mayor tasa de recurrancias se encuentran: un estadío tumor primario T2 o mayor, invasión linfovascular y/o hallazgo histológico de predominio de carcinoma de células embrionarias (4).

Después del abordaje multidisciplinario del teratoma testicular metastásico avanzado, la tasa de supervivencia sin enfermedad es excelente, y aproximadamente el $90 \%$ de los pacientes permanece libre de enfermedad de forma contínua (4).

\section{CONCLUSIÓN}

En la medida de los posible debemos tratar el quiste dermoide testicular de la forma menos agresiva posible, ya que se trata de un tumor con muy baja capacidad metastásica. Se recomienda el tratamiento quirúrgico conservador con excisión local y biopsias testiculares como ocurre en el caso de los quistes epidermoides. Sin embargo no está claro que el quiste dermoide sea tan claramente identificable en la ecografía, como en el caso del quiste epidermoide, y que permitiera su diagnóstico preoperatorio. Por ello, este tratamiento conservador, sólo debe realizarse en aquellos casos en los que la conservación testicular sea esencial (2). 


\section{BIBLIOGRAFÍA Y LECTURAS RECOMENDADAS (*lectura de interés $y^{* *}$ lectura fundamental)}

1. Leibovitch I, Foster RS, Ulbright TM, Donohue JP. Adult primary pure teratoma of the testis. The Indiana experience. Cancer, 1995; 75:2244-2250.

**2. Ulbright TM, Srigley JR. Dermoid Cyst of the testis. A study of five postpubertal cases, including a pilomatrixoma-like variant, with evidence supporting its separate classification from mature testicular teratoma. Am. J. Surg. Pathol. 2001; 25(6):788-793.

3. Young RH. Testicular tumors-some new and a few prennial problems. Arch. Pathol. Lab. Med. 2008; 132:548-564.

*4. Carver BS, Al-Ahmadie H, Sheinfeld J. Teratoma testicular adulto y pediátrico. Urol. Clin. N. Am. 2007; 34:245-251.

5. Vázquez Rueda F, Moñiz Mora MV, Salas Molina J, Pizarro de Celis FJ, Ocaña Losa JM. Teratoma testicular y coristoma adrenal epididimario. Actas Urol. Esp. 2000; 24(4):351-4.

6. Nistal N, González-Peramato P, Regadera J. Anatomia patológica de las neoplasias testiculares. Tratado de Urología Oncológica. Resel Estévez L, Moreno Sierra J. Ed: Sanidad y Ediciones (Saned). 2003; 2(75):977-996.

*7. Ulbright TM, Amin MB, Young RH. Tumor of the testis, adnexa, spermatic cord, and scrotum. Atlas of tumor pathology. 3rd series Washington DC. Armed Forces Institute of Pathology Fas. 25. 1999.

8. Royal SA, Joseph DB, Galliani CA. Bilateral testicular teratoma. AJR. 1994; 163:1130.

9. Rabbani F, Farivar-Mosheni H, León A, Motzer RJ, Bosl GJ, Sheinfeld J. Clinical outcome after retroperitoneal lymphadenectomy of patients with pure testicular teratoma. Urology, 2003; 62:1092-6.

*10. Porcaro AB, Antoniolli SZ, Martignoni G, Brunelli, Curti P. Adult primary teratoma of the testis-report on 5 cases in clinical stage I disease. Int. J. Urol. Nephrol. 2001; 33:657-9.

11. Heidenreich A, Moul JW, McLeod DG, Mostofi FK, Engelmann UH. The role of retroperitoneal lymphadenectomy in mature teratoma of the testis. J. Urol. 1997; 157:160-3.

12. Moul JW, McCarthy WF, Fernández EB, Sesterhenn IA. Percentage of embryonal carcinoma and of vascular invasion predicts pathological stage in clinical stage I nonseminomatous testicular cancer. Cancer Res. 1994; 54:362-4.

**13. Minkowitz G, Lee M, Minkowitz S. Pilomatricoma of the testicle: an ossifying testicular tumor with hair matrix differentiation. Arch. Pathol. Lab. Med. 1995; 119:96-9.

14. Serrano P, Fantova A, Pascual M, Allué M, Gil MJ, Rioja LA. Tratamiento del teratoma maduro puro de testículo metastatizado ¿Qué hacer? Arch. Esp. Urol. 2006; 59(5):524-6. 